\title{
GANGRENA SIMÉTRICA PERIFÉRICA (GSP): RELATO DE CASO E REVISÃO NARRATIVA DA LITERATURA
}

\section{PERIPHERAL SYMMETRICAL GANGRENA (GSP): CASE REPORT AND NARRATIVE LITERATURE REVIEW}

Gustavo Lopes Gomes de Siqueira ${ }^{1}$; Ana Beatriz de Lima Borba ${ }^{1}$; Nicolle Mabel de Almeida Vieira ${ }^{1}$; Raquel Duarte Rolim ${ }^{1}$.

\section{RESUMO}

Introdução: A gangrena simétrica periférica (GSP) é uma desordem microvascular caracterizada por isquemia e gangrena majoritariamente nos membros inferiores, sendo intimamente relacionada com quadros de coagulação intravascular disseminada (CIVD) e choque. Consiste numa síndrome rara, porém comumente confundida na prática médica com a má perfusão pelo uso de drogas vasoativas (DVA). Relato do caso: É feito o relato do caso de um paciente de 1 ano e 11 meses, sem antecedentes patológicos relevantes, acometido por metapneumovirose que evoluiu com choque séptico e CIVD. No segundo dia de internação, foi realizado o diagnóstico de GSP em membros inferiores e superiores. Conclusão: Discute-se o papel dos vasopressores na sindrome, sua fisiopatologia e as principais opções terapêuticas para esses casos.

Palavras-chave: Gangrena. Criança. Vasoconstritores.

\section{ABSTRACT}

Introduction: Peripheral symmetric gangrene (GSP) is a microvasculature disorder characterized by ischemia and gangrene mostly in the lower limbs and deeply related to disseminated intravascular coagulation (DIC) and shock. It is a rare syndrome but commonly confused with the poor perfusion caused by the use of vasoactive drugs (VAD). Case report: This case reports a patient aged 1 year and 11 months with no relevant pathological history affected by a metapneumovirus with septic shock and severe thrombocytopenia. On the second day of hospitalization he was diagnosed with GSP in the lower limbs. Conclusion: It is discussed the role of vasopressors agents in the syndrome, its pathophysiology and the main therapeutic options for these cases.

Keywords: Gangrene. Child. Vasoconstrictor Agents.

\section{INTRODUÇÃO}

A gangrena simétrica periférica (GSP) se caracteriza por isquemia/gangrena acral de dois ou mais membros, com ausência de obstrução macrovascular. A maioria dos casos reportados ocorre em pacientes com desordem inflamatória importante, condições críticas que levem à coagulação intravascular disseminada (CIVD) e desequilíbrio entre fatores pró e anticoagulantes, culminando em trombose de microvasculatura ${ }^{1}$.

A metapneumovirose humana é causada por vírus de ácido ribonucleico (RNA), sendo descrito pela primeira vez em 2001. Estudos demostraram que a virose apresenta sazonalidade e quadro clínico mais grave em lactentes e na primeira idade de vida, com pico de hospitalização entre 6 e 12 meses. Sua prevalência em lactentes internados com infecção aguda respiratória é de $6,24 \%$ e estudos soroepidemiológicos demonstram que até $100 \%$ dos adultos tiveram contato com o virus ${ }^{2,3}$.

O objetivo deste trabalho é descrever a experiencia com caso único de GSP, em criança com sindrome do desconforto respiratório agudo por metapenumovirose, contribuindo para o melhor entendimento de uma patologia rara e comumente confundida com uso de drogas vasoativas ou outro tipos de trombose arterial, expandindo o conhecimento através de comparação e revisão da literatura. 


\section{RELATO DO CASO}

Paciente do sexo masculino, 1 ano e 11 meses, com quadro de sintomas gripais típicos por 10 dias, evidenciando piora nos últimos 3 dias antes da internação, com aumento da frequência respiratória e febre. Apresentava história prévia médica sua e familiar irrelevante.

Ao exame fisico, apresentava taquipneia, mesmo em uso de oxigênio por máscara inalatória, com $32 \mathrm{ipm}$, e taquicardia, com $147 \mathrm{bpm}$. Iniciou-se, de forma empírica, ceftriaxona (100mg/kg/dia), oxacilina $(300 \mathrm{mg} / \mathrm{kg} / \mathrm{dia})$ e oseltamivir (30mg/ $\mathrm{kg} /$ dose). Evoluiu com agitação e sinais de sindrome do desconforto respiratório agudo, assim como hipotensão (pressão arterial de 68 x $37 \mathrm{mmHg}$. Realizou-se, então, intubação orotraqueal cerca de 12 horas após a admissão, com expansão volêmica (10ml/kg em 1 hora). Como não se obteve resposta satisfatória, iniciou-se adrenalina $(0,15 \mathrm{mcg} / \mathrm{kg} / \mathrm{min})$ e dobutamina $(7,5 \mathrm{mcg} / \mathrm{kg} / \mathrm{min})$ endovenosa. Os exames laboratoriais apresentavam, à entrada: leucograma de $21,5 \times 10^{3} / \mathrm{mm}^{3}$ com $5,0 \%$ de bastões, plaquetas de $168 \times 10^{3} / \mathrm{mm}^{3}$; função renal e coagulograma normais; proteína $\mathrm{C}$ reativa (PCR) quantitativa de $96 \mathrm{mg} / \mathrm{dl}$. Realizou-se tomografia de tórax com imagem em vidro fosco bilateral e áreas com infiltrado parenquimatoso. A ultrassonografia abdominal total indicou nefropatia parequimatosa.

Diagnosticou-se choque séptico por sindrome do desconforto respiratório agudo e optou-se por trocar antibioticoterapia para cobertura de germes atípicos com tazocin $(300 \mathrm{mg} / \mathrm{kg} /$ dia) e vancomicina $(60 \mathrm{mg} / \mathrm{kg} / \mathrm{dia})$, mantendo o oseltamivir (30mg/dose).

No segundo dia de internação iniciou-se um quadro de cianose fixa acral em todos os pododáctilos e quirodáctilos, com área de cianose não fixa (zona de penumbra) em região metacarpiana e metatarsiana (Figura 1). Apresentava pulsos radiais normais associado a fluxo trifásico ao Ultrassom Color Doppler. Nos membros inferiores, o indice tornozelobraço (ITB) apresentava-se dentro da normalidade. Como não havia sinais de infecção local, optou-se por aquecimento local e manutenção de suporte cardiovascular.

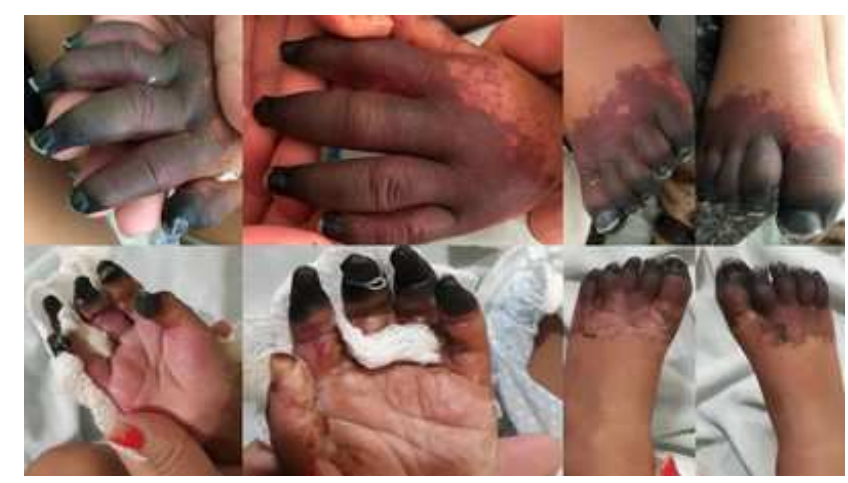

Figura 1: Quadro inicial: na parte superior segundo dia de internação com zona de penumbra extensa e área de necrose pequena distal. Na parte inferior no dia 17 de internação com delimitação parcial.

\section{Os exames demonstraram} plaquetopenia severa $\left(48 \times 10^{3} / \mathrm{mm}^{3}\right)$, atividade de protrombina reduzida $(57,9 \%)$ e international normalized ratio (INR) levemente alargado $(1,67)$. Os exames de função hepática estavam normais.

Fez-se uso de vasopressores nos dois primeiros dias, com adrenalina $(0,15 \mathrm{mcg} / \mathrm{kg} / \mathrm{min})$ e dobutamina $(7,5 \mathrm{mcg} / \mathrm{kg} / \mathrm{min})$. A partir do terceiro dia, utilizou-se somente dobutamina, com redução progressiva, por mais cinco dias.

$O$ resultado do swab nasal para pesquisa viral foi positivo para metapneumovirose. A hemocultura foi negativa.

Evoluiu, nos dias seguintes, com sinais de disfunção renal aguda leve, apresentando pico de ureia $(71 \mathrm{mg} / \mathrm{dl})$ e creatinina $(1,5 \mathrm{mg} / \mathrm{dl})$ no quarto dia de internação. Com relação à CIVD, apresentou alargamento do INR (pico de 1,81 , com atividade de protrombina de $20 \%$ no quarto dia de internação) e plaquetopenia (pico de $41 \times 10^{3} / \mathrm{mm}^{3}$ no quarto dia de internação), sendo diagnóstico para CIVD tanto nos critérios da Sepsis-induced coagulopathy (SIC) quanto Japanese Association for Acute Medicine (JAAM) score systems (maior ou igual à 4 pontos nos dois escores) ${ }^{4}$.

Com tratamento clínico de suporte hemodinâmico e antibioticoterapia, apresentou melhora dos parâmetros respiratórios e foi submetido à extubação orotraqueal no sétimo dia de internação hospitalar. No $11^{\circ}$ dia de internação, apresentou pico febril $\left(38^{\circ} \mathrm{C}\right)$. Como era paciente ainda em uso de máscara de oxigênio, aventou-se a hipótese de 
pneumonia bacteriana e trocou-se o antibiótico por meropenem $(120 \mathrm{mg} / \mathrm{kg} / \mathrm{dia})$. Apresentou melhora evidente nos dias subsequentes, tendo alta da unidade de terapia intensiva (UTI) para enfermaria no $14^{\circ}$ dia de internação hospitalar.

Com relação ao quadro de GSP, optou-se por observação clínica dos segmentos acometidos até a delimitação mais precisa das áreas de necrose (Figura 1). Sendo paciente submetido à amputação, no $29^{\circ}$ dia de internação, de todos os pododáctilos e de falanges distais de quirodáctilos à direita, bem como amputação de falanges distais dos quirodáctilos esquerdos (exceto polegar, que não apresentava sinais de isquemia). Observou-se cicatrização completa e normal das feridas operatórias (Figuras 2 e 3).

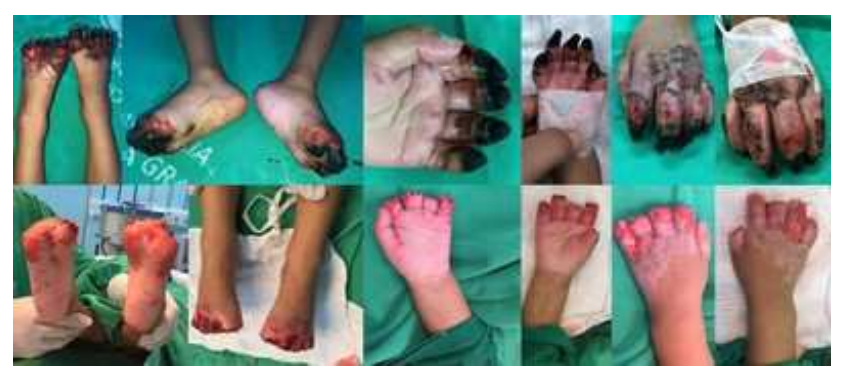

Figura 2: Vigésimo nono dia de internação: área de necrose já delimitada no pré-operatório. $\mathrm{Na}$ parte inferior o pós-operatório imediato.

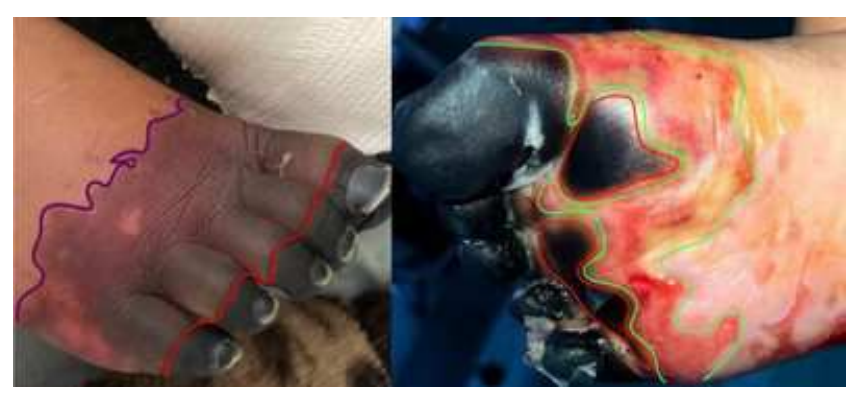

Figura 3: Na figura à direita a isquemia em fase aguda, com a linha roxa delimitando a zona de penumbra e a linha vermelha a zona de gangrena. $\mathrm{Na}$ figura à esquerda a fase delimitada com a linha vermelha a área de gangrena e a verde a zona de hiperemia (recuperação da isquemia).

\section{DISCUSSÃO}

A GSP é uma síndrome rara, definida por isquemia acral simétrica de dois ou mais segmentos, com evidente ausência de oclusão arterial da macrovasculatura (pulsos presentes distalmente). Sua frequência é controversa na literatura, acometendo, aproximadamente, 2\% dos pacientes críticos com choque refratário. Localiza-se, na grande maioria, nos membros inferiores, e é possível que ocorra o acometimento associado dos membros superiores. Como a fisiopatologia demanda trombose da microvasculatura, as lesões ocorrem principalmente na falange distal, e dependendo da gravidade, todo o dedo. A disfunção da coagulação se deve a um desequilíbrio por consumo de fatores anticoagulantes naturais e ativação inapropriada da trombina, com consequente trombose da microvasculatura. Três principais características estão sempre presentes: (1) choque circulatório, com microtrombose, poupando macrovasculatura; (2) CIVD; e (3) profundo consumo de anticoagulantes naturais. Grande parcela evolui com "choque hepático", mediante aumento das transaminases e depleção de fatores anticoagulantes de produção hepática (proteína $\mathrm{C}$, principalmente). Uma descrição de série de 15 casos reportou elevação das transaminases em 14 pacientes, com depleção de proteínas C e S em 100\% dos casos $^{5}$.

O quadro clínico geralmente evidencia uma associação de estado geral grave com choque circulatório, sendo comum que emergencistas e intensivistas confundam sua fisiopatologia com o uso de drogas vasoativas (DVA). A isquemia iniciase distalmente, sendo identificada por cianose fixa, bolhas hemorrágicas, e proximalmente por zonas de cianose não fixa (zona de penumbra). Posteriormente, com a melhora hemodinâmica, advém a área de hiperemia e redução, em geral, da zona de penumbra6.

$\mathrm{O}$ quadro laboratorial geralmente apresenta cenário de CIVD com trombocitopenia, aumento de fibrinogênio e d-dimero ${ }^{7}$.

Grande parte dos pacientes apresenta (90\%) choque hepático, com hepatite isquêmica geralmente precedendo o quadro, em média, três dias, explicando o porquê de a síndrome ocorrer com depleção de anticoagulantes naturais 5,8 . A histopatologia da GSP é corroborada por deposição trombótica de fibrina dos pequenos vasos, devido ao desequilíbrio entre fatores pró e anticoagulantes ${ }^{9}$. 
No caso descrito, a criança deu entrada no hospital já em choque séptico refratário a volume, iniciando-se vasopressores. Já no primeiro dia, apresentou plaquetopenia $\left(108 \times 10^{3} / \mathrm{mm}^{3}\right)$, precedendo o quadro de isquemia periférica que se iniciou dois dias após a entrada. Embora não apresentava sinais de hepatite isquêmica, ao longo da internação apresentou disfunção de múltiplos órgãos (respiratório e renal), assim como plaquetopenia severa $\left(41 \mathrm{x} \quad 10^{3} / \mathrm{mm}^{3}\right)$ e alteração da coagulação (INR de 1,81), sendo diagnóstico para $\mathrm{CIVD}^{4}$, com pico no quarto dia. Não existe nível de evidência forte $\mathrm{e}$ independente ligando $\mathrm{o}$ uso de vasopressores com a isquemia acral ${ }^{10}$.

Vários autores não encontraram débito cardíaco baixo e resistência vascular elevada em demasia no uso de vasopressores na sindrome ${ }^{11,12}$. Em revisão narrativa de casos de GSP com CIVD, nenhum estava usando vasopressor no momento do início do quadro isquêmico ${ }^{13}$. Uma revisão de estudos randomizados publicados pela Cochrane ${ }^{14}$ não encontrou indícios correlacionando eventos isquêmicos acrais e o uso dos seis vasopressores. Outro fator importante é que o quadro isquêmico acral na GSP, em série de casos estudados, só se desenvolveu, em média, três dias (dois a cinco dias) após o início do quadro de choque circulatório refratário e o uso de DVA, tempo demais para se estabelecer correlação direta ${ }^{3,5}$. Em revisão sobre o papel dos vasopressores e a isquemia de membros, observaram-se somente oito estudos com bons critérios. A conclusão foi de que o aparecimento da GSP se devia mais ao quadro de sepse profundamente grave e coagulopatia, do que pelo uso dos vasopressores, citando que há uma inabilidade atual para referenciar a isquemia acral com o uso de drogas vasoativas ${ }^{10}$. Um estudo retrospectivo casocontrole sobre uso de DVA e GSP demonstrou que a duração do uso de DVA não influencia o aparecimento de gangrena, mas o pico da dose pode ter papel de relevância no surgimento de gangrena, principalmente para dopamina e noradrenalina ${ }^{14}$. Em trabalhos descritivos de casos de GSP na literatura, os pacientes nem chegaram a fazer uso de DVA para estabelecer correlação entre causa e efeito ${ }^{16,17}$. Uma revisão sobre o assunto não encontrou diferença das doses e a duração de uso de DVA entre pacientes sem e com GSP18. Esperar-se-ia que, se o papel das DVA na sindrome fosse predominante, os pacientes que evoluíssem com isquemia acral deveriam usar doses maiores, o que não aconteceu de fato. Diante de várias evidências; alguns pacientes descritos com GSP sequer usaram DVA; não foi encontrado aumento de resistência vascular periférica; a dose e duração de uso dos vasopressores não foi diferente; a literatura se torna clara quanto à falta de correlação entre o uso de DVA e o quadro de GSP. Isso não acontece com o quadro de coagulopatia e CVID, estando sempre presente, aceitando-se, por isso, um desequilíbrio entre fatores pró e anticoagulantes como substrato fisiopatológico principal, com depleção de trombina em microcirculação, mantendo a macrovasculatura pérvia, com pulsos distais palpáveis. No caso descrito, a criança iniciou o uso de DVA com adrenalina $\quad(0,15 \mathrm{mcg} / \mathrm{kg} / \mathrm{min}) \quad$ e dobutamina $(7,5 \mathrm{mcg} / \mathrm{kg} / \mathrm{min})$ nos dois primeiros dias, e do terceiro ao sétimo dia utilizou somente dobutamina. O início dos vasopressores precedeu o quadro de isquemia acral (GSP), em dois dias, estando de acordo com a literatura 3,5 . O uso dos vasopressores não pareceu ter papel predominante no quadro descrito, pois, no início do quadro isquêmico, o paciente já estava em franca retirada da adrenalina (manteve somente a dobutamina), sem relação imediata de causa e efeito, diferentemente da CIVD, que teve papel evidente, com coincidência entre sua ascensão e piora do quadro de isquemia.

O manejo da GSP se relaciona muito com o manejo da sepse grave. O consenso sobre o manejo da sepse não indica utilização de heparinizacão plena para pacientes com CIVD ${ }^{19,20}$, exceto aqueles com diagnóstico de trombose de médios e grandes vasos. O tratamento principal reside na remoção do fator causal, prevenção de infecção secundária e suporte hemodinâmico. Concentrados de antitrombina e de trombomodulina foram propostos no início do milênio, mas estudos subsequentes não mostraram melhora clinica ${ }^{20-22 .}$

É importante o manejo dos membros, com aquecimento frouxo e monitorização quanto ao aparecimento de 
complicações, principalmente síndrome compartimental, que pode demandar cirurgia de emergência. Como a mortalidade na fase aguda dos pacientes com GSP pode chegar a $40 \% \%^{3,5,6,10}$, não é fidedigno o indice de amputação, que só deve ser realizada na presença de infecção ou gangrena bem delimitada. Reportaramse beneficios com o uso de antagonistas alfa-adrenérgicos (clorpromazina ou fentolamina) e de vasodilatadores mediante a inibição da fosfodiesterase, mas sem estudos prospectivos 6,23 . Em média, deve-se esperar um longo tempo de observação, algumas séries de casos citaram meses 5,6,10, outras, em média, quatro semanas ${ }^{5,8}$ para a realização da retirada do tecido desvitalizado, podendo ou não levar à amputação. No caso descrito, a criança foi submetida a amputação de pododáctilos e quirodáctilos (Figura 2). No presente caso, evidenciou-se que a observação e espera da delimitação da área necrótica resultou em cirurgia de menor dano.

\section{REFERÊNCIAS}

1. Warkentin TE. Microvascular Thrombosis and Ischaemic Limb Losses in Critically Ill Patients. Hamostaseologie. 2019;39(1):6-19.

doi: $10.1055 / \mathrm{s}-0038-1676823$.

2. Ravindranath TM, Gomez A, Harwayne-Gidansky I, Connors TJ, Neill $\mathrm{N}$, Levin B, et al. Pediatric acute respiratory distress syndrome associated with human metapneumovirus and respiratory syncytial virus. Pediatr Pulmonol. 2018;53(7):929-35.

doi: $10.1002 /$ ppul.24044.

3. Lefebvre A, Manoha C, Bour JB, Abbas R, Fournel I, Tiv M, et al. Human metapneumovirus in patients hospitalized with acute respiratory infections: A metaanalysis. J Clin Virol. 2016;81:68-77.

doi: $10.1016 / j . j c v .2016 .05 .015$.

4. Iba T, Umemura $Y$, Watanabe $E$, et al. Diagnosis of sepsis-induced disseminated intravascular coagulation and coagulopathy. Acute Med Surg. 2019;6(3):223-32.

doi: $10.1002 /$ ams2.411.

5. Warkentin TE. Ischemic limb gangrene with pulses. N Engl J Med. 2015;373(7):642-55.

doi: 10.1056/NEJMra1316259.
6. Shenoy R, Agarwal N, Goneppanavar U, Shenoy A, Sharma A. Symmetrical peripheral gangrene-a case report and brief review. Indian J Surg. 2013;75(Suppl 1):163-5. doi: $10.1007 / \mathrm{s} 12262-012-0576-7$.

7. Hayakawa M, Gando S, Ono Y, Wada T, Yanagida Y, Sawamura A. Fibrinogen level deteriorates before other routine coagulation parameters and massive transfusion in the early phase of severe trauma: a retrospective observational study. Semin Thromb $\mathrm{He}-$ most. 2015;41(1):35-42.

doi: 10.1055/s-0034-1398379.

8. Warkentin TE. Anticoagulant failure in coagulopathic patients: PTT confounding and other pitfalls. Expert Opin Drug Saf. 2014;13(01):25-43. doi: $10.1517 / 14740338.2013 .823946$.

9. Moake JL. Disseminated Intravascular coagulation (DIC) (Consumption Coagulopathy; Defibrination Syndrome). MSD Manual Professional Version. 2020.

10. Levy JH, Ghadimi K, Faraoni D, van Diepen S, Levy B, Hotchkiss R, et al. Ischemic limb necrosis in septic shock: What is the role of high-dose vasopressor therapy. J Thromb Haemost. 2019;17(11):1973-8.

doi: $10.1111 /$ jth. 14566 .

11. Hayes MA, Yau EHS, Hinds CJ, Watson JD. Symmetrical peripheral gangrene: association with noradrenaline administration. Intensive Care Med. 1992;18(7):433-6.

doi: $10.1007 /$ BF01694349.

12. Joynt G, Doedens L, Lipman J, Bothma P. High-dose adrenaline with low systemic vascular resistance and symmetrical peripheral gangrene. S Afr J Surg. 1996;34(2):99-101.

13. Ghosh SK, Bandyopadhyay D, Ghosh A. Symmetrical peripheral gangrene: a prospective study of 14 consecutive cases in a tertiary-care hospital in eastern India. J Eur Acad Dermatol Venereol. 2010;24(2):214-8. doi: 10.1111/j.1468-3083.2009.03329.x.

14. Gamper G, Havel C, Arrich J, Losert H, Pace NL, Müllner M, et al. Vasopressors for hypotensive shock. Cochrane Database Syst Rev. 2016;2(2):CD003709. doi: 10.1002/14651858.CD003709.pub4. 
15. Kwon JW, Hong MK, Park BY. Risk Factors of Vasopressor-Induced Symmetrical Peripheral Gangrene. Ann Plast Surg. 2018;80(6):622-7. doi: 10.1097/SAP.0000000000001314.

16. Agrawal A, Rastogi A, Tiwari D. Symmetric peripheral gangrene with mixed malaria. Indian J Pediatr. 2007 Jun;74(6):587-8. doi: 10.1007/s12098-007-0100-4.

17. Kurup V, Simpson RS. Sepsis-induced digital ischaemia in a professional pianist, in the absence of vasopressors. BMJ Case Rep. 2019;12(10):e229659. doi: 10.1136/bcr2019-229659.

18. Warkentin TE, Ning S. Symmetrical peripheral gangrene in critical illness. Transfus Apher Sci. 2021;60(2): 103094.

doi: 10.1016/j.transci.2021.103094.

19. Rhodes A, Evans LE, Alhazzani W, Levy MM, Antonelli M, Ferrer R, et al. Surviving Sepsis Campaign: international guidelines for management of sepsis and septic shock: 2016. Intensive Care Med 2017;43(3):304-77. doi: $10.1007 / \mathrm{s} 00134-017-4683-6$.

20. Zarychanski R, Abou-Setta AM, Kanji S, Turgeon AF, Kumar A, Houston DS, et al. Canadian Critical Care Trials Group. The efficacy and safety of heparin in patients with sepsis: a systematic review and metaanalysis. Crit Care Med. 2015;43(3):511-8. doi: 10.1097/CCM.0000000000000763.

21. Allingstrup M, Wetterslev J, Ravn FB, Møller AM, Afshari A. Antithrombin III for critically ill patients. Cochrane Database Syst Rev. 2016;2(2):CD005370. doi:

10.1002/14651858.CD005370.pub3.

22. Ranieri VM, Thompson BT, Barie PS, Dhainaut JF, Douglas IS, Finfer S, et al. Drotrecogin alfa (activated) in adults with septic shock. N Engl J Med. 2012;366(22):2055-64. doi: 10.1056/NEJMoa1202290.

23. Kaul S, Sarela AI, Supe AN, Karnard DR. Gangrene complicating dopamine therapy. J R Soc Med. 1997;90(2):80. doi: $10.1177 / 014107689709000207$.
Fonte de financiamento: Não

Conflito de interesses: Não

Data de Submissão: 11 Maio 2021

Decisão final: 22 Novembro 2021

\section{Autor de Correspondência:}

Gustavo Lopes Gomes de Siqueira

E-mail: gustavomed@yahoo.com 